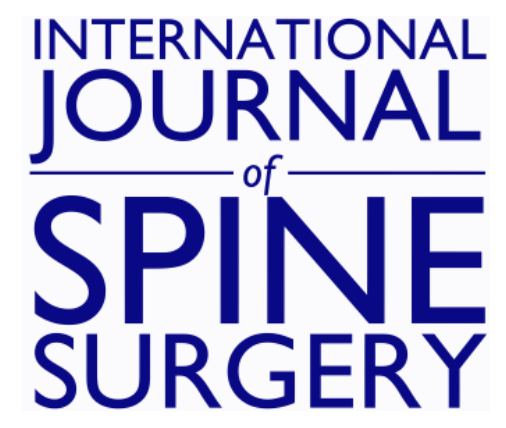

\title{
Video-assisted thoracoscopic surgery as a diagnostic and therapeutic instrument in non-tubercular spondylodiscitis
}

Deniece Détillon, Hans de Groot, Eric Hoebink, Rob Versteylen and Eelco Veen

Int J Spine Surg 2015, 9 ()

doi: https://doi.org/10.14444/2055

http://ijssurgery.com/content/9/55

This information is current as of April 26, 2023.

Email Alerts Receive free email-alerts when new articles cite this article. Sign up at:

http://ijssurgery.com/alerts

The International Journal of SWing Surgerth 2397 Waterbury Circle, Suite 1,

Aurora, IL 60504, Phone: +1-630-375-1432

(C) 2015 ISASS. All Rights Reserved. 


\section{Video-assisted thoracoscopic surgery as a diagnostic and therapeutic instrument in non-tubercular spondylodiscitis}

Deniece Détillon, ${ }^{1}$ Hans de Groot, $M D,{ }^{1}$ Eric Hoebink, $M D,{ }^{2}$ Rob Versteylen, $M D,{ }^{3}$ Eelco Veen, $M D, P h D^{1}$

${ }^{1}$ Department of Surgery, Amphia Hospital, Breda, Netherlands ${ }^{2}$ Department of Orthopaedic Surgery, Amphia Hospital, Breda, Netherlands ${ }^{3}$ Department of Radiology, Amphia Hospital, Breda, Netherlands

\section{Abstract}

Background

Spondylodiscitis refers to an infection of one or more intervertebral disks and vertebrae, most commonly caused by tuberculosis. Initial therapy for spondylodiscitis is drug treatment. Indications for surgical treatment include compression of neural elements, spinal instability or severe deformities. When surgery is indicated, an open technique is still the standard, although video-assisted thoracoscopic surgery (VATS) is described in cases of thoracic disk herniation, scoliosis and tubercular spondylitis.

\section{Case report}

We report a case of a 79-year-old man with progressive back pain, paraparesis and paresthesias in both legs. During assessment, spondylodiscitis with spinal cord compression was diagnosed. Immediate surgical decompression took place by means of VATS. Culture of the tissue obtained revealed Proteus mirabilis. Blood and urine cultures also revealed Proteus mirabilis, a rare cause of spondylodiscitis with an occurrence rate of $0.9 \%$.

\section{Conclusion}

VATS offered a minimally invasive access to obtain the diagnosis of pyogenic spondylodiscitis following urosepsis with Proteus mirabilis. Moreover, it provided treatment with abscess drainage and decompression of the spinal cord.

KEYWORDS: VIDEO-ASSISTED THORACOSCOPIC SURGERY, SPONDYLODISCITIS, THORACIC SPINE, PROTEUS MIRABILIS

\section{Introduction}

Spondylodiscitis refers to an infection of one or more intervertebral disks and vertebrae, being aetiologically granulomatous, pyogenic or parasitic. Of the granulomatous infections, tuberculosis is the most common cause of spondylodiscitis. Staphylococus aureus is the most common pathogen in pyogenic spondylodiscitis, followed by Enterobacteria. Parasitic infections are uncommon. ${ }^{1}$

Initial therapy is drug treatment. Surgical treatment is indicated for spinal instability with severe deformities, or if conservative treatment does not improve the patients' condition. Spinal cord compression requires immediate surgical decompression. ${ }^{1}$

Video-assisted thoracoscopic surgery (VATS) for the treatment of spinal disorders has been used mostly in cases of thoracic disk herniation, scoliosis ${ }^{2}$ and tu- bercular spondylitis. ${ }^{2,3}$ There are no previous reports about the use and efficacy of VATS as a diagnostic and therapeutic instrument in non-tubercular spondylodiscitis, which will be discussed in this case report.

\section{Case presentation}

A 79-year-old man presented to his general practitioner with a two-month history of back pain. X-rays were performed and revealed decreased bone density of the vertebrae, loss of definition of the caudal endplate of T10 and the cranial endplate of T11 with a wedge-shaped compression and formation of a block vertebra. Eight days later he was admitted for progressive back pain, paraparesis and paresthesias in both legs. He has had recurrent urinary tract infections and bladder stones, and was treated with lithotripsy and transurethral resection of the prostate two weeks prior to admission. 
A new X-ray of the thoracic spine showed a soft tissue shadow at the T10-T11 level. The clinical presentation, combined with the X-ray was suspected for spondylodiscitis. Magnetic resonance imaging (MRI) and computed tomography (CT) confirmed the radiological diagnosis of spondylodiscitis (Figure 1 and Figure 2). Because of the impending paraplegia an immediate surgical decompression took place.

A right-sided VATS procedure for definitive diagnosis and treatment was performed. Under general anaesthesia, an endobronchial blocker was inserted and the patient was placed in left lateral position. After selective collapse of the right lung, the camera was inserted at the level of the 11th intercostal space. A utility incision $(2-3 \mathrm{~cm})$ was made ventral of the patient at the 5 th intercostal space and a wound retractor was placed for easy access. Inspection showed bulging of the parietal pleura without contamination of the pleural cavity (Figure 3). The abscess was punctured with an endoscopic needle, which showed purulent fluid. Afterwards, the parietal pleura was opened resulting in sufficient drainage of the abscess.

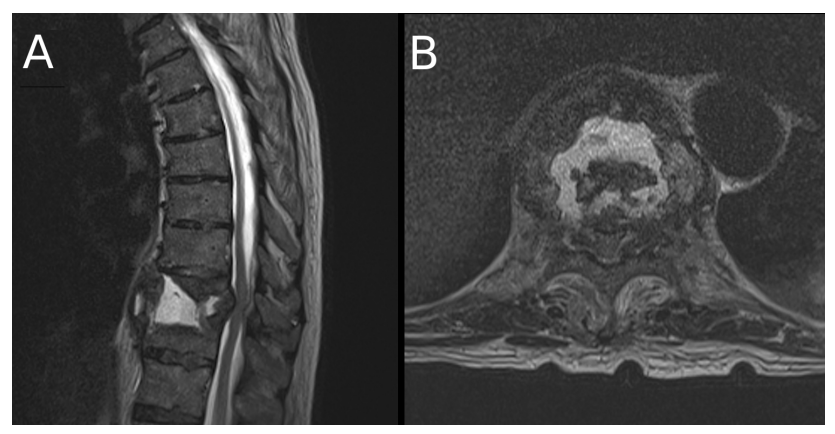

Fig. 1. MRI of thoracic spine, sagittal ( $A$ ) and transversal (B), showing destruction of the T10-T11 vertebrae and spinal cord compression.
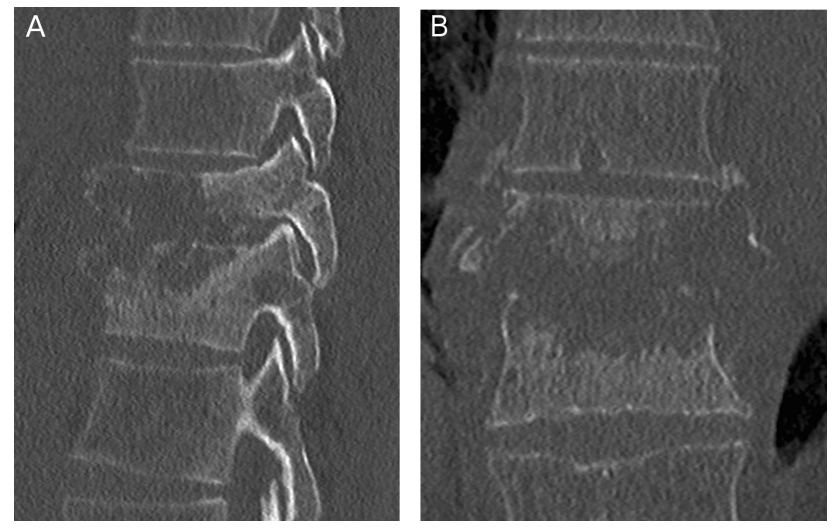

Fig. 2. CT of thoracic spine, sagittal (A) and coronal (B), showing bone destruction of the T10-T11 vertebrae.
After the abscess was drained adequately a debridement was performed with biopsies taken of the pleura and the abscess.

The patient was kept one day in the intensive care unit for observation and was nursed completely immobilized because of spinal instability. Postoperatively there were no signs of paraplegia. After two days, additional posterior percutaneous stabilization was performed, to stabilize the spine.

Blood and tissue cultures revealed Proteus mirabilis. Urine cultures, conducted two weeks prior to admission, also revealed Proteus mirabilis. The patient tested negative for tuberculosis. Pathologists confirmed the diagnosis of infection without signs of malignancy. The diagnosis of spondylodiscitis following urosepsis with Proteus mirabilis was therefore made. Intravenous antibiotic treatment with ceftriaxone was commenced, for the duration of six weeks. Both postoperative recoveries were uneventful and the patient was discharged from hospital 15 days after initial surgery.

At eleven-month follow-up the patients is able to walk without devices and increased bone density without recurrence of spondylodiscitis was seen on $\mathrm{CT}$ and X-rays.

\section{Discussion}

Spondylodiscitis can be classified as pyogenic, granulomatous (tuberculosis, brucellosis, fungal) or parasitic. ${ }^{1,4}$ The pathogenesis of pyogenic spondylodiscitis can be haematogenous, by external inoculation or

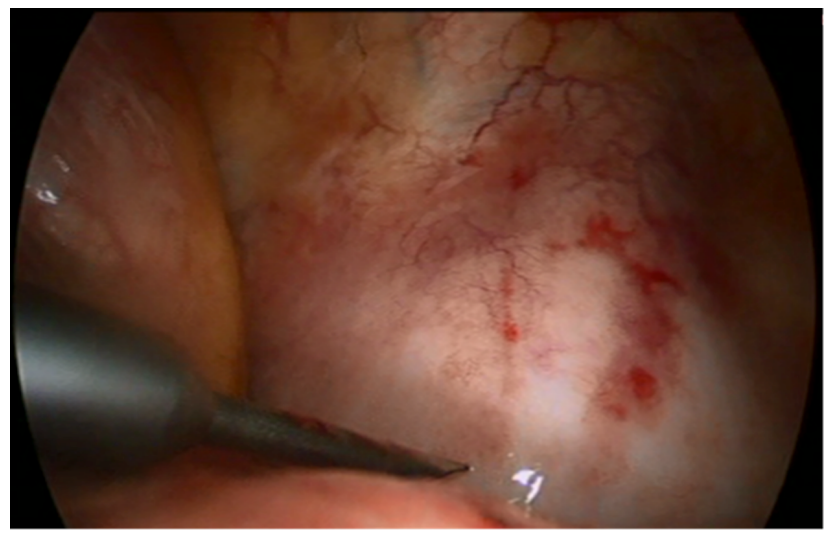

Fig. 3. Intra-operative view of the bulging parietal pleura. 
by spread from contiguous tissues. ${ }^{1}$ Haematogenous pyogenic spondylodiscitis is predominant, ${ }^{1}$ with Staphylococcus aureus as the most common causative agent, ${ }^{1,4}$ followed by enterobacteria. ${ }^{1}$ Pseudomona aureaginosa is often seen in intravenous drug users. Also Coagulase negative staphylococci, Streptococci and Enterococci have been described. ${ }^{1}$ Proteus mirabilis is less common and was found in $0.9 \%$ of cases. ${ }^{4}$

X-rays may be useful if spondylodiscitis is suspected. However, large destructive spondylodiscitis is not always recognized on X-rays as changes may take months to appear. Moreover, it can be difficult to distinguish infection from degenerative changes. ${ }^{1}$ This is evident in the present case; the abnormalities seen on X-rays eight days prior to admission were probably an indication of spondylodiscitis. Gadoliniumenhanced MRI is the imaging modality of choice for diagnosing spondylodiscitis. CT is the best imaging modality to delineate areas of bone destruction, which is useful for surgical planning. ${ }^{1}$

Initial therapy for spondylodiscitis is drug treatment. Also when surgery is indicated, additional drug treatment is essential. Indications for surgical treatment include compression of neural elements, spinal instability due to bone destruction, severe deformities such as kyphosis or if conservative treatment does not improve the patients' condition. The presence of spinal cord compression is an indication for immediate surgical decompression. ${ }^{1}$ However, if a patient is paralyzed preoperatively, surgical decompression is unlikely to undo paralysis. ${ }^{4}$

Open surgery is nowadays still the standard, particularly in cases of large bone destruction. ${ }^{1}$ However, indications for VATS are similar to those requiring open techniques. VATS can be used when lesions involve the anterior spinal column, from T2-L1. ${ }^{3}$ The VATS procedure has been described in literature mostly in cases of thoracic disk herniation and scoliosis. ${ }^{2}$ VATS can also be a useful alternative to conventional thoracotomy in tubercular thoracic spondylitis. ${ }^{2,3}$ However, thoracoscopy is not indicated for all patients. The contraindications for this technique are patients' intolerance to one-lung ventilation or severe pleural adhesions. ${ }^{2,3}$
Major advantages of VATS are the reduction of intraoperative blood loss, shorter postoperative stay and cosmetically appealing minor surgical scars along with less postoperative pain, when compared with thoracotomy. ${ }^{2}$

In this study, VATS offered a minimally invasive access to obtain the diagnosis of pyogenic spondylodiscitis and provided treatment with abscess drainage and decompression of the spinal cord. VATS can be considered as a valuable diagnostic and therapeutic instrument for spondylodiscitis, due to its minimal invasive approach.

\section{References}

1. Guerado E, Cervan AM. Surgical treatment of spondylodiscitis. an update. Int Orthop.

2012;36(2):413-420.

2. Kapoor SK, Agarwal PN, Jain BK,Jr, Kumar R. Video-assisted thoracoscopic decompression of tubercular spondylitis: Clinical evaluation. Spine (Phila Pa 1976). 2005;30(20):E605-10.

3. Huang TJ, Hsu RW, Chen SH, Liu HP. Videoassisted thoracoscopic surgery in managing tuberculous spondylitis. Clin Orthop Relat Res.

2000;(379)(379):143-153.

4. Hadjipavlou AG, Mader JT, Necessary JT, Muffoletto AJ. Hematogenous pyogenic spinal infections and their surgical management. Spine (Phila Pa 1976). 2000;25(13):1668-1679.

\section{Disclosures}

The authors declare no relevant disclosures.

\section{Corresponding author}

Deniece Détillon, Department of Surgery, Amphia Hospital, Molengracht 21, 4818 CK Breda, Netherlands. Tel: +31 765951151; Fax: +31 765953818; email: DDetillon@amphia.nl.

Published 26 October 2015.

This manuscript is generously published free of charge by ISASS, the International Society for the Advancement of Spine Surgery. Copyright @ 2015 
ISASS. To see more or order reprints or permissions, see http://ijssurgery.com. 